\title{
Optimization of Continuous-Time Observations for the Stationary Kalman-Bucy Filter
}

\author{
Yoshiki TAKEUCHI \\ Dept. of Information Science, Osaka Kyoiku University \\ 4-698-1 Asahigaoka, Kashiwara City, Osaka 582-8582, Japan \\ Tel. +81-729-78-3669, Fax. +81-729-78-3554 \\ e-mail: takeuti@cc.osaka-kyoiku.ac.jp
}

\begin{abstract}
In this paper, we are concerned with a problem of optimization of the linear observations which are used in the stationary Kalman-Bucy filter. Especially, we consider the optimization of the gain matrix in the observation. In the previous works of the author, the corresponding problem for discrete-time systems was already considered and the condition of optimality was obtained. This paper is concerned with the case of the continuous-time systems and it is shown that the condition of optimality is the same as the discrete-time case except for the accompanying Lyapunov equation is continuous-type whereas it was discrete-type in the discrete-time problem. We propose a method of solving the set of equations of the Riccati equation for the error covariance and the condition of optimality by a simple recursive algorithm. The results of numerical experiments show the efficiency of the algorithm.
\end{abstract}

\section{Introduction}

In this paper, we consider an optimization problem for the gain matrix of a linear observation which is used for the stationary Kalman-Bucy filter. The criterion of the optimization is given by a sum of the weighted variance of the estimation errors and a quadratic function of the gain matrix. In previous works of the author, the discrete-time problem was considered [7]-[13], and in [13], the following results have been obtained.

(1) The optimal value of the observation matrix is not unique, but we can obtain one of them from the optimal value of the Symmetric Signal-to-Noise Ratio Matrix (SSNRM) which is a quadratic function of the gain matrix weighted by the noise covariance;

(2) The original problem, with the observation gain matrix as the variable, is converted to an optimization problem with variables of a pair of matrices (one with the set of eigenvectors and the other diagonal matrix with the eigenvalues of SSNRM);

(3) The condition of optimality is obtained in the form that each eigenvector of SSNRM should be also an eigenvector of a symmetric matrix which is linearly dependent on a solution of a matrix Lyapunov equation.

Also, numerical algorithms to compute the optimal values were developed by using general purpose mathematical nonlinear programing tools.

In this paper, we will first show the results corresponding to (1)-(3):

(1)' We can obtain an optimal value of the gain matrix from that of the same SSNRM as in the discrete-time case;

(2)' The original problem is converted to an optimization problem with variables of the same pair of matrices as in the discrete-time case;

(3)' The condition of optimality is obtained in the same form as in the discrete-time case except for the point that the accompanying Lyapunov equation becomes continuous-type.

Then, we apply the fact:

(4)' The optimization problem is with the variables:

(a) the pair of matrices obtained in (2);

(b) the error covariance matrix;

(c) the symmetric matrix which is given by a solution of the Lyapunov equation obtained in (3)'; and is concerned with the equations:

(x) the condition of optimality with (b) and (c);

(y) the Lyapunov equation with (a), (b) and (c);

(z) the Riccati equation of the error covariance with (a) and (b);

in order to show the following fact:

(5)' In the special case where the dimension of the observation is equal to that of the signal and where all the components of the observation noise are mutually independent, we can obtain an equation with only (b) as the variable. Then, the solution process is straightforward;

(6)' In the case where the dimension of the observation is equal to that of the signal, we have the same-type of equation as (5)' but including (b) and (c) in its coefficients. We can construct a recursive algorithm with $(\mathrm{x})$ and $(\mathrm{z})$ together with this, and can easily compute the solution of the set of equations.

The results of the numerical computation for illustrative examples are shown for better understanding of the result of this paper.

Mathematical symbols, in this paper, are used in the following way. $\boldsymbol{R}$ is the space of all real numbers, i.e., $\boldsymbol{R} \triangleq(-\infty, \infty)$. For positive integers $m$ and $n, \boldsymbol{R}^{n}$ and $\boldsymbol{R}^{m \times n}$ denote the spaces of $n$-dimensional vectors and 
$m \times n$-dimensional matrices whose components take values in $\boldsymbol{R}$. The prime denotes the transpose of a vector or a matrix and the Euclidean norm is $|\cdot|$. Thus, for $x \in \boldsymbol{R}^{n}, \quad|x|=\sqrt{x^{\prime} x}$. The identity matrix of any dimension is denoted by $I$. The components of a matrix are denoted by using subscripts. Thus, $[A]_{i j}$ is the $(i, j)$-component of $A$. In the case where no confusion may arise, we denote $[A]_{i j}$ simply by $a_{i j}$. If $A$ is a square matrix, $\operatorname{det}|A|$ and $\operatorname{tr}[A]$ respectively denote the determinant and the trace of $A$. We use $A>0$ and $A \geq 0$ to denote that $A$ is positive definite and nonnegative definite, respectively. For any pair of matrices $A$ and $B, A \otimes B$ denotes the Kronecker product of $A$ and $B$, and $\operatorname{vec}(A)$ is the vector formed by stacking the columns of $A$ into a single column vector. The triplet $(\Omega, \mathscr{F}, P)$ is a complete probability space where $\Omega$ is a sample space with elementary events $\omega, \mathscr{F}$ is a $\sigma$-field of subsets of $\Omega$, and $P$ is a probability measure. $E\{\cdot\}$ denotes the expectation and $E\{\cdot \mid \mathscr{G}\}, \mathscr{G} \subset \mathscr{F}$ the conditional expectation, given $\mathscr{G}$, with respect to $P$. $\sigma\{\cdot\}$ is the minimal sub- $\sigma$-field of $\mathscr{F}$ with respect to which the family of $\mathscr{\mathscr { F }}$-measurable sets or random variables $\{\cdot\}$ is measurable.

\section{Problem Formulation}

Let $\boldsymbol{x} \equiv\left\{x_{t}(\omega) ; 0 \leq t<\infty\right\}$ be an $n$-dimensional Gaussian stochastic process generated by the linear system:

$$
\left\{\begin{aligned}
d x_{t}(\omega) & =A x_{t}(\omega) d t+G d w_{t}(\omega) \\
x_{0}(\omega) & =x^{0}(\omega)
\end{aligned}\right.
$$

where $A \in \boldsymbol{R}^{n \times n}, G \in \boldsymbol{R}^{n \times d_{1}}, x^{0}(\omega)$ is a Gaussian random vector with mean $\hat{x}^{0}$ and covariance $Q^{0}$, and $\boldsymbol{w} \equiv$ $\left\{w_{t}(\omega) ; 0 \leq t<\infty\right\}$ is a $d_{1}$-dimensional standard Brownian motion process. Suppose that the value of $\boldsymbol{x}$ is not directly available but we have linear observations described by

$$
d y_{t}(\omega)=H x_{t}(\omega) d t+R d v_{t}(\omega)
$$

where $\boldsymbol{y} \equiv\left\{y_{t}(\omega) ; 0 \leq t<\infty\right\} \quad$ is an $m$-dimensional observation process, $H \in \boldsymbol{R}^{m \times n}, \quad R \in \boldsymbol{R}^{m \times d_{2}}$, and $\boldsymbol{v} \equiv$ $\left\{v_{t}(\omega) ; 0 \leq t<\infty\right\}$ is a $d_{2}$-dimensional standard Brownian motion process. Throughout this paper, we will assume that the following two conditions are satisfied.

(C-1) $R_{0} \triangleq R R^{\prime}>0$,

(C-2) $x^{0}(\omega), \boldsymbol{w}$ and $\boldsymbol{v}$ are mutually independent.

It is well-known that the least-squares estimate $\hat{x}_{t}(\omega) \triangleq$ $E\left\{x_{t}(\omega) \mid \mathscr{Y}_{t}\right\} \quad$ of $\quad x_{t}(\omega)$ based on $\mathscr{Y}_{t} \triangleq \sigma\left\{y_{s}(\omega)\right.$; $0 \leq s \leq t\}$ is given by the Kalman-Bucy:

$$
\left\{\begin{aligned}
d \hat{x}_{t}(\omega) & =A \hat{x}_{t}(\omega) d t+Q_{t} H^{\prime} R_{0}^{-1}\left\{d y_{t}(\omega)-H \hat{x}_{t}(\omega) d t\right\} \\
\hat{x}_{0}(\omega) & =E\left\{x^{0}(\omega)\right\},
\end{aligned}\right.
$$

and

$$
\left\{\begin{aligned}
\frac{d Q_{t}}{d t} & =A Q_{t}+Q_{t} A^{\prime}+G G^{\prime}-Q_{t} H^{\prime} R_{0}^{-1} H Q_{t} \\
Q_{0} & =E\left\{\left[x_{0}(\omega)-\hat{x}_{0}(\omega)\right]\left[x_{0}(\omega)-\hat{x}_{0}(\omega)\right]^{\prime}\right\},
\end{aligned}\right.
$$

where

$$
Q_{t} \triangleq E\left\{\left[x_{t}(\omega)-\hat{x}_{t}(\omega)\right]\left[x_{t}(\omega)-\hat{x}_{t}(\omega)\right]^{\prime}\right\},
$$

and

$$
R_{0} \triangleq R R^{\prime} .
$$

In this paper, we are concerned with the stationary Kalman-Bucy filter in which, under the assumption for (4) that $Q_{t} \rightarrow Q$ as $t \rightarrow \infty$, we have, instead of (3) and (4), the following equations:

$$
\begin{aligned}
& \left\{\begin{aligned}
d \hat{x}_{t}(\omega) & =A \hat{x}_{t}(\omega) d t+Q H^{\prime} R_{0}^{-1}\left\{d y_{t}(\omega)-H \hat{x}_{t}(\omega) d t\right\} \\
\hat{x}_{0}(\omega) & =E\left\{x^{0}(\omega)\right\},
\end{aligned}\right. \\
& A Q+Q A^{\prime}+G G^{\prime}-Q H^{\prime} R_{0}^{-1} H Q=0 .
\end{aligned}
$$

For the optimization of performance of the least-squares state estimator (7) and (8), we introduce the following performance criterion: ${ }^{[13]}$

$$
J \triangleq \operatorname{tr}[M Q]+\operatorname{tr}\left[H N H^{\prime}\right],
$$

where $\operatorname{tr}[M Q]$ is an weighted estimation error variance, i.e.,

$$
\operatorname{tr}[M Q]=E\left\{\left[x_{t}(\omega)-\hat{x}_{t}(\omega)\right]^{\prime} M\left[x_{t}(\omega)-\hat{x}_{t}(\omega)\right]\right\},
$$

and $M \in \boldsymbol{R}^{n \times n}$ and $N \in \boldsymbol{R}^{r \times r}$ are positive-definite symmetric matrices. In (9), the second term in the right-hand side denotes the cost or the energy consumed by the observation.

Thus, we are now concerned with the following problem.

[Problem 1] Find $H \in \boldsymbol{R}^{m \times n}$ such that (9) is mini- mized subject to (8).

The mathematical treatment and result of Problem 1 are very similar to those of the discrete-time problems previously discussed in Takeuchi [13-17].

The key idea of the approach is an application of the orthonormal-diagonal representation of the symmetric matrix $N^{1 / 2} H^{\prime} R_{0}^{-1} H N^{1 / 2}$. Note that this symmetric matrix is linearly dependent on $H^{\prime} R_{0}^{-1} H$ which may be called a symmetric signal-to-noise ratio matrix (SSNRM). Then, the variable $H \in \boldsymbol{R}^{m \times n}$, the observation gain matrix, is replaced by a more convenient set of variables in finding the minimal value of (9). Then, we can easily obtain an optimal value of $H$ which is, in general, not necessarily unique in the space $\boldsymbol{R}^{m \times n}$.

Since $\quad N^{1 / 2} H^{\prime} R_{0}^{-1} H N^{1 / 2}$ is a nonnegative-definite symmetric matrix, we can represent this matrix as

$$
N^{1 / 2} H^{\prime} R_{0}^{-1} H N^{1 / 2}=\tilde{U} \tilde{\Xi} \tilde{U}^{\prime},
$$

where $\tilde{U}=\left[u_{1} u_{2} \cdots u_{\tilde{m}}\right] \in \boldsymbol{R}^{n \times \tilde{m}}$ is the set of eigenvectors of $N^{1 / 2} H^{\prime} R_{0}{ }^{-1} H N^{1 / 2}$ corresponding to the positive eigenvalues $\xi_{i}, i=1,2, \cdots, \tilde{m}$, and

$$
\begin{gathered}
\tilde{\Xi} \triangleq \operatorname{diag}\left(\xi_{1}, \xi_{2}, \cdots, \xi_{\tilde{m}}\right), \quad \xi_{i}>0, i=1,2, \cdots, \tilde{m}, \\
\tilde{m} \triangleq \operatorname{rank}[H](\leq m) .
\end{gathered}
$$

Without loss of generality, we can assume that

$$
\xi_{1} \geq \xi_{2} \geq \cdots \geq \xi_{\tilde{m}}>0 .
$$


For any $\tilde{U} \in \boldsymbol{R}^{n \times \tilde{m}}$ with property $\tilde{U}^{\prime} \tilde{U}=I$ and any $\tilde{\Xi}$ with properties (12) and (13), let

$$
\mathscr{H}(\tilde{U}, \tilde{\Xi}) \triangleq\left\{H \in \boldsymbol{R}^{m \times n} ; N^{1 / 2} H^{\prime} R_{0}^{-1} H N^{1 / 2}=\tilde{U} \tilde{\Xi} \tilde{U}^{\prime}\right\} .
$$

Thus, $\mathscr{H}(\tilde{U}, \tilde{\Xi})$ is the set of values of $H$ for which $N^{1 / 2} H^{\prime} R_{0}^{-1} H N^{1 / 2}$ has fixed positive eigenvalues $\xi_{i}$ and the corresponding eigenvectors $u_{i}, i=1,2, \cdots, \tilde{m}$.

From (11), we have

$$
H^{\prime} R_{0}{ }^{-1} H=N^{-1 / 2} \tilde{U} \tilde{\Xi} \tilde{U}^{\prime} N^{-1 / 2}, \quad H \in \mathscr{H}(\tilde{U}, \tilde{\Xi}) .
$$

Now, we have the following theorem which guarantees that we can always take $H$ in the form (17) given below.

«Theorem 1»[13]-[17] Assume (C-1) and (C-2). Then, we have the following properties.

(i) $Q$, the solution of (8), is completely determined by $\left(A, G G^{\prime}, \tilde{U}, \tilde{\Xi}, N\right)$.

(ii) For a fixed set of value $(\tilde{U}, \tilde{\Xi})$ and for any $H \in \mathscr{H}(\tilde{U}, \tilde{\Xi})$, we have the same value of $Q$.

(iii) For any set of value $(\tilde{U}, \tilde{\Xi})$, a set of elements in $\mathscr{H}(\tilde{U}, \tilde{\Xi})$ is always given by

$$
H=R_{0}{ }^{1 / 2} \tilde{\Gamma} \tilde{\Xi}^{1 / 2} \tilde{U}^{\prime} N^{-1 / 2},
$$

where $\tilde{\Gamma} \in \boldsymbol{R}^{m \times \tilde{m}}$ denotes the first $\tilde{m}$ columns of an orthogonal matrix $\Gamma \in \boldsymbol{R}^{m \times m}$ such that $\Gamma \Gamma^{\prime}=\Gamma^{\prime} \Gamma=I$.

(iv) At least, one of the optimal values of $H \in \boldsymbol{R}^{m \times n}$ is given by the form (17).

(Proof) We have the assertions immediately by noting the fact that $Q$ is determined by (8) with (16).

Thus, without loss of generality, we can take $H$ as the form given by (17) which is an expression of $H$ with property (16). Thus, the problem has been converted to the one of the optimization with respect to $\tilde{\Gamma} \in \boldsymbol{R}^{m \times \tilde{m}}$, $\tilde{U} \in \boldsymbol{R}^{n \times \tilde{m}}$ and $\tilde{\Xi} \triangleq \operatorname{diag}\left(\xi_{1}, \xi_{2}, \cdots, \xi_{\tilde{m}}\right)$.

[Remark 1] Theorem 1 and (16) imply that the optimal value of $H$ may not be unique but there are a number of minimum points in the space $\boldsymbol{R}^{m \times n}$ of $H$, and that by the change of variables $H \rightarrow(\tilde{U}, \tilde{\Xi})$, these minimum points are mapped into a single point in the space of $(\tilde{U}, \tilde{\Xi})$. This is a main reason that the method of optimization of $H$ given in this paper is efficient.

\section{The Condition of Optimality}

As we see from (8), (16) and (17), the value of $Q$ is independent of that of $\tilde{\Gamma} \in \boldsymbol{R}^{m \times \tilde{m}}$. Hence, the optimal value of $\tilde{\Gamma}$ should be determined in such a way that $\operatorname{tr}\left[H H^{\prime}\right]$, the second term in (9), is minimized. This minimization is done by the exactly same way as the discrete-time case.

«Theorem 2» ${ }^{[13]}$ Assume (C-1)-(C-2). Then the optimal value of $\tilde{\Gamma} \in \boldsymbol{R}^{m \times \tilde{m}}$ is given by the set of eigenvectors of $R_{0}$ corresponding to the first $\tilde{m}$ eigenvalues in ascending order, i.e., we have the relation

$$
\begin{gathered}
R_{0}=\left[\begin{array}{cc}
\tilde{\Gamma} & \bar{\Gamma}
\end{array}\right] \Psi\left[\begin{array}{c}
\tilde{\Gamma}^{\prime} \\
\bar{\Gamma}^{\prime}
\end{array}\right], \quad \Psi=\operatorname{diag}\left(\psi_{1}, \psi_{2}, \cdots, \psi_{\tilde{m}}, \cdots, \psi_{m}\right), \\
\psi_{1} \leq \psi_{2} \leq \cdots \leq \psi_{\tilde{m}} \leq \cdots \leq \psi_{m} .
\end{gathered}
$$

[Remark 2] If we take $\tilde{\Gamma} \in \boldsymbol{R}^{m \times \tilde{m}}$ according to Theorem 2, we have

where

$$
H=\tilde{\Gamma} \tilde{\Psi}^{1 / 2} \tilde{\Xi}^{1 / 2} \tilde{U}^{\prime} N^{-1 / 2},
$$

$$
\tilde{\Psi} \triangleq \operatorname{diag}\left(\psi_{1}, \psi_{2}, \cdots, \psi_{\tilde{m}}\right) .
$$

[Remark 3] For the existence and the uniqueness of nonnegative-definite and positive-definite solutions of (8), explicit conditions are known ([18; Sec. 3.5], [19]).

Now, we have converted Problem 1 to the following form.

[Problem 2] Find $\tilde{U} \in \boldsymbol{R}^{n \times \tilde{m}}$ and $\tilde{\Xi}=\operatorname{diag}\left(\xi_{1}, \xi_{2}, \cdots\right.$, $\left.\xi_{\tilde{m}}\right)$ such that

$$
J=\operatorname{tr}[M Q]+\operatorname{tr}[\tilde{\Psi} \tilde{\Xi}] \rightarrow \min .,
$$

subject to (8), (16), (14) and

$$
\tilde{U}^{\prime} \tilde{U}=I \text {. }
$$

For Problem 2, let us define the Lagrangean by

$$
L(\tilde{\Xi}, \tilde{U}, \tilde{\Lambda}) \triangleq \operatorname{tr}[M Q]+\operatorname{tr}[\tilde{\Psi} \tilde{\Xi}]+\operatorname{tr}\left[\tilde{\Lambda}\left(\tilde{U}^{\prime} \tilde{U}-I\right)\right],
$$

where $\tilde{\Lambda} \in \boldsymbol{R}^{\tilde{m} \times \tilde{m}}$ is a symmetric matrix whose $(i, j)$ component is a Lagrange multiplier for the same component of (22), i.e.,

$$
\begin{aligned}
\operatorname{tr}\left[\tilde{\Lambda}\left(\tilde{U}^{\prime} \tilde{U}-I\right)\right] & =\sum_{i=1}^{\tilde{m}} \sum_{j=1}^{\tilde{m}} \lambda_{i j}\left[\left(\tilde{U}^{\prime} \tilde{U}-I\right)\right]_{j i} \\
& =\sum_{i=1}^{\tilde{m}} \sum_{j=1}^{\tilde{m}} \lambda_{i j}\left[\left(\tilde{U}^{\prime} \tilde{U}-I\right)\right]_{i j}
\end{aligned}
$$

For Problem 2, we have the following result.

«Theorem 3 (Condition of Optimality)» Let us define $F \in \boldsymbol{R}^{n \times n}$ by

$$
F \triangleq A-Q N^{-1 / 2} \tilde{U} \tilde{\Xi} \tilde{U}^{\prime} N^{-1 / 2}
$$

Assume (C-1), (C-2) and

(C-3) The set of $\tilde{U}$, determined by

$$
\mathscr{D}(A) \triangleq\left\{\tilde{U} \in \boldsymbol{R}^{n \times \tilde{m}} ; \tilde{U}^{\prime} \tilde{U}=I,\left(\tilde{U}^{\prime}, A\right) \text { is obserbable }\right\},
$$

is not empty. Also, the optimal value of $\tilde{U}$ belongs to $\mathscr{D}(A)$.

Then, the condition of optimality of $\tilde{U}$ and $\tilde{\Xi}$ is given by

$$
N^{-1 / 2} Q X Q N^{-1 / 2} \tilde{U}=\tilde{U} \tilde{\Psi},
$$

where $X \in \boldsymbol{R}^{n \times n}$ is a solution of

$$
F^{\prime} X+X F+M=0 \text {. }
$$

Since $\tilde{\Psi}$ is a diagonal matrix given by (20), (26) 
implies that

«Corollary $1 »$ Assume (C-1)-(C-3). The optimal $(\tilde{U}, \tilde{\Xi})$ is such that

(i) Each column vector of $\tilde{U}$ is an eigenvector of $N^{-1 / 2} Q X Q N^{-1 / 2}$ which is a symmetric matrix.

(ii) The order of column vectors in $\tilde{U}$ is the one that the corresponding eigenvalues are in ascending order.

(iii) The $\tilde{m}$ eigenvalues of $N^{-1 / 2} Q X Q N^{-1 / 2}$ that corresponds to $\tilde{U}$ coincide with the first $\tilde{m}$ eigenvalues of $R_{0}$.

For the proof of Theorem 3, we need the following lemma.

«Lemma 1» Assume (C-1)-(C-3). The solutions of matrix Lyapunov equations:

$$
F^{\prime} X+X F+Z=0
$$

and

$$
F \hat{X}+\hat{X} F^{\prime}+\hat{Z}=0,
$$

are given respectively by

$$
\operatorname{vec}(X)=-\left(F^{\prime} \otimes I+I \otimes F^{\prime}\right)^{-1} \operatorname{vec}(Z),
$$

and

$$
\operatorname{vec}(\hat{X})=-(F \otimes I+I \otimes F)^{-1} \operatorname{vec}(\hat{Z}) .
$$

(Proof) By the well-known property vec $(X Y Z)=$ $\left(Z^{\prime} \otimes X\right) \operatorname{vec}(Y)$ of the Kronecker product and the relations: $F^{\prime} X=F^{\prime} X I$ and $X F=I X F,(28)$ can be represented by

$$
\left(F^{\prime} \otimes I+I \otimes F^{\prime}\right) \operatorname{vec}(X)=-\operatorname{vec}(Z),
$$

and which implies (30). Since we have (29) from (28) if we replace $F, X$ and $Z$ in (28) by $F^{\prime}, \hat{X}$ and $\hat{Z}$ respectively, we see that the solution of (29) is given by (31). This completes the proof.

(Proof of Theorem 3) From (8) and (16), we have

$$
\begin{aligned}
& A \frac{\partial Q}{\partial u_{i j}}+\frac{\partial Q}{\partial u_{i j}} A^{\prime}-\frac{\partial Q}{\partial u_{i j}} N^{-1 / 2} \tilde{U} \tilde{\Xi} \tilde{U}^{\prime} N^{-1 / 2} Q \\
& -Q N^{-1 / 2} \tilde{U} \tilde{\Xi} \tilde{U}^{\prime} N^{-1 / 2} \frac{\partial Q}{\partial u_{i j}} \\
& -Q N^{-1 / 2}\left\{E_{i j} \tilde{\Xi} \tilde{U}^{\prime}+\tilde{U} \tilde{\Xi} E_{j i}\right\} N^{-1 / 2} Q \\
& =\left\{A-Q N^{-1 / 2} \tilde{U} \tilde{\Xi} \tilde{U}^{\prime} N^{-1 / 2}\right\} \frac{\partial Q}{\partial u_{i j}} \\
& +\frac{\partial Q}{\partial u_{i j}}\left\{A-Q N^{-1 / 2} \tilde{U} \tilde{\Xi} \tilde{U}^{\prime} N^{-1 / 2}\right\}^{\prime} \\
& -Q N^{-1 / 2}\left\{E_{i j} \tilde{\Xi} \tilde{U}^{\prime}+\tilde{U} \tilde{\Xi} E_{j i}\right\} N^{-1 / 2} Q=0,
\end{aligned}
$$

where

$$
E_{i j} \triangleq\left[\begin{array}{ccc}
0 & 0 & 0 \\
& \vdots & 0 \\
0 \cdots 0 & 1 & 0 \cdots 0 \\
0 & 0 & 0
\end{array}\right] i,
$$

Thus, noting (25), the derivative $\partial Q / \partial u_{i j}$ is given by the solution of the Lyapunov equation:

$$
\begin{aligned}
& F \frac{\partial Q}{\partial u_{i j}}+\frac{\partial Q}{\partial u_{i j}} F^{\prime} \\
& -Q N^{-1 / 2}\left\{E_{i j} \tilde{\Xi} \tilde{U}^{\prime}+\tilde{U} \tilde{\Xi} E_{j i}\right\} N^{-1 / 2} Q=0 .
\end{aligned}
$$

By (29) and (31) of Lemma 1, we have

$$
\begin{gathered}
\operatorname{vec}\left(\frac{\partial Q}{\partial u_{i j}}\right)=(F \otimes I+I \otimes F)^{-1} \operatorname{vec}(S), \\
S \triangleq Q N^{-1 / 2}\left\{E_{i j} \tilde{\Xi} \tilde{U}^{\prime}+\tilde{U} \tilde{\Xi} E_{j i}\right\} N^{-1 / 2} Q .
\end{gathered}
$$

Now, from (23), we have

$\frac{\partial}{\partial u_{i j}} L(\tilde{\Xi}, \tilde{U}, \tilde{\Lambda})=\operatorname{tr}\left[M \frac{\partial Q}{\partial u_{i j}}\right]+\operatorname{tr}\left[\tilde{\Lambda}\left\{E_{j i} \tilde{U}+\tilde{U}^{\prime} E_{i j}\right\}\right]$.

By noting the relations $\operatorname{tr}[A B]=\operatorname{vec}\left(A^{\prime}\right)^{\prime} \operatorname{vec}(B)$ and $(A \otimes B)^{\prime}=A^{\prime} \otimes B^{\prime}$, we see that

$$
\begin{aligned}
\operatorname{tr}\left[M \frac{\partial Q}{\partial u_{i j}}\right] & =\operatorname{vec}(M)^{\prime} \operatorname{vec}\left(\frac{\partial Q}{\partial u_{i j}}\right) \\
& =\operatorname{vec}(M)^{\prime}\left\{(F \otimes I+I \otimes F)^{-1} \operatorname{vec}(S)\right\} \\
& =\left\{(F \otimes I+I \otimes F)^{\prime-1} \operatorname{vec}(M)\right\}^{\prime} \operatorname{vec}(S) \\
& =\left\{\left(F^{\prime} \otimes I+I \otimes F^{\prime}\right)^{-1} \operatorname{vec}(M)\right\}^{\prime} \operatorname{vec}(S) \\
& =-\operatorname{vec}(X)^{\prime} \operatorname{vec}(S) \\
& =\operatorname{tr}[X S],
\end{aligned}
$$

where $X \in \boldsymbol{R}^{n \times n}$ is the solution of (27). Substituting (37) into the last expression in (39), we have

$$
\begin{aligned}
\operatorname{tr}\left[M \frac{\partial Q}{\partial u_{i j}}\right]= & -\operatorname{tr}\left[X Q N^{-1 / 2}\left\{E_{i j} \tilde{\Xi} \tilde{U^{\prime}}+\tilde{U} \tilde{\Xi} E_{j i}\right\} N^{-1 / 2} Q\right] \\
= & -\operatorname{tr}\left[N^{-1 / 2} Q X Q N^{-1 / 2}\left\{E_{i j} \tilde{\Xi} \tilde{U^{\prime}}+\tilde{U} \tilde{\Xi} E_{j i}\right\}\right] \\
= & -\operatorname{tr}\left[E_{i j} \tilde{\Xi} \tilde{U}^{\prime} N^{-1 / 2} Q X Q N^{-1 / 2}\right] \\
& -\operatorname{tr}\left[N^{-1 / 2} Q X Q N^{-1 / 2} \tilde{U} \tilde{\Xi} E_{j i}\right] \\
= & -2 \operatorname{tr}\left[N^{-1 / 2} Q X Q N^{-1 / 2} \tilde{U} \tilde{\Xi} E_{j i}\right] \\
= & -2\left[N^{-1 / 2} Q X Q N^{-1 / 2} \tilde{U} \tilde{\Xi}\right]_{i j} .
\end{aligned}
$$

Since the last term in the right-hand side of (38) is computed as

$$
\begin{aligned}
\operatorname{tr}\left[\tilde{\Lambda}\left\{E_{j i} \tilde{U}+\tilde{U}^{\prime} E_{i j}\right\}\right] & =2 \operatorname{tr}\left[\tilde{U} \tilde{\Lambda} E_{j i}\right] \\
& =2[\tilde{U} \tilde{\Lambda}]_{i j},
\end{aligned}
$$

the condition of optimality is obtained from the relation $\left(\partial / \partial u_{i j}\right) L(\tilde{\Xi}, \tilde{U}, \tilde{\Lambda})=0$ with (38), (40) and (41) as 


$$
\left[N^{-1 / 2} Q X Q N^{-1 / 2} \tilde{U} \tilde{\Xi}\right]_{i j}=[\tilde{U} \tilde{\Lambda}]_{i j},
$$

or equivalently,

$$
N^{-1 / 2} Q X Q N^{-1 / 2} \tilde{U} \tilde{\Xi}=\tilde{U} \tilde{\Lambda} .
$$

On the other hand, the same way of computation for $\left(\partial / \partial \xi_{i}\right) L(\tilde{\Xi}, \tilde{U}, \tilde{\Lambda})$ is done as follows. From (8), we have

$$
F \frac{\partial Q}{\partial \xi_{i}}+\frac{\partial Q}{\partial \xi_{i}} F^{\prime}-Q N^{-1 / 2} \tilde{U} E_{i i} \tilde{U}^{\prime} N^{-1 / 2} Q=0 .
$$

Hence, we have

$$
\begin{aligned}
\operatorname{vec}\left(\frac{\partial Q}{\partial \xi_{i}}\right) & =(F \otimes I+I \otimes F)^{-1} \operatorname{vec}(\tilde{S}), \\
\tilde{S} & \triangleq Q N^{-1 / 2} \tilde{U} E_{i i} \tilde{U}^{\prime} N^{-1 / 2} Q .
\end{aligned}
$$

Then, it follows that

$$
\begin{aligned}
\operatorname{tr}\left[M \frac{\partial Q}{\partial \xi_{i}}\right] & =\operatorname{vec}(M)^{\prime} \operatorname{vec}\left(\frac{\partial Q}{\partial \xi_{i}}\right) \\
& =-\operatorname{vec}(X)^{\prime} \operatorname{vec}(\tilde{S}) \\
& =-\left[\tilde{U}^{\prime} N^{-1 / 2} Q X Q N^{-1 / 2} \tilde{U}\right]_{i i},
\end{aligned}
$$

where $X \in \boldsymbol{R}^{n \times n}$ is the solution of (27). The condition of optimality is obtained from the relation $\left(\partial / \partial \xi_{i}\right) L(\tilde{\Xi}, \tilde{U}, \tilde{\Lambda})=0$ with (23), (47) and

$$
\frac{\partial}{\partial \xi_{i}} \operatorname{tr}[\tilde{\Psi} \tilde{\Xi}]=\operatorname{tr}\left[\tilde{\Psi} E_{i i}\right]=[\tilde{\Psi}]_{i i},
$$

as

$$
\left[\tilde{U}^{\prime} N^{-1 / 2} Q X Q N^{-1 / 2} \tilde{U}\right]_{i i}=[\tilde{\Psi}]_{i i},
$$

or equivalently,

$$
\tilde{U}^{\prime} N^{-1 / 2} Q X Q N^{-1 / 2} \tilde{U}=\tilde{\Psi} .
$$

Now, because of (22), (43) multiplied by $\tilde{U}^{\prime}$ from left is represented by

$$
\tilde{U}^{\prime} N^{-1 / 2} Q X Q N^{-1 / 2} \tilde{U} \tilde{\Xi}=\tilde{\Lambda} .
$$

Substitution of (50) into (51) yields

$$
\tilde{\Psi} \tilde{\Xi}=\tilde{\Lambda} \text {. }
$$

From (43), (52) and $\tilde{\Xi}>0$, we have (26) for $X \in \boldsymbol{R}^{n \times n}$ which is a solution of (27). This completes the proof.

[Remark 4] The condition (26) of the optimality is exactly the same as the one in the discrete-time case [13] except for the point that $X \in \boldsymbol{R}^{n \times n}$ in this case is a solution of the continuous-type Lyapunov equation whereas it is a solution of the discrete-type Lyapunov equation in the discrete-time case.

\section{Solution of the Problem}

By Theorem 3, the solution of Problem 2 is given by a set of equations for $(Q, \tilde{U}, \tilde{\Xi}, X)$ :

$$
\begin{gathered}
A Q+Q A^{\prime}+G G^{\prime}-Q N^{-1 / 2} \tilde{U} \tilde{\Xi} \tilde{U}^{\prime} N^{-1 / 2} Q=0, \\
N^{-1 / 2} Q X Q N^{-1 / 2} \tilde{U}=\tilde{U} \tilde{\Psi},
\end{gathered}
$$

$$
\begin{gathered}
F^{\prime} X+X F+M=0, \\
F \triangleq A-Q N^{-1 / 2} \tilde{U} \tilde{\Xi} \tilde{U}^{\prime} N^{-1 / 2},
\end{gathered}
$$

with the constraints:

$$
\tilde{U}^{\prime} \tilde{U}=I
$$

and

$$
\xi_{1} \geq \xi_{2} \geq \cdots \geq \xi_{\tilde{m}}>0 .
$$

In fact, the optimal observation gain matrix is given by (19) if we obtain the solution $(Q, \tilde{U}, \tilde{\Xi}, X)$ of the above set of equations. In this section, methods of solving this set of equations will be developed.

First, let us show that (53) and (25)-(27) are converted into an equivalent more convenient form.

«Theorem 4 » Assume (C-1)-(C-3). The set of equations: (53) and (25)-(27) are equivalent to

$$
\begin{gathered}
\left(A+G G^{\prime} Q^{-1}\right) Q X Q+Q X Q\left(A+G G^{\prime} Q^{-1}\right)^{\prime}-Q M Q=0,(54) \\
Q X Q=N^{1 / 2}\left\{\tilde{U} \tilde{\Psi} \tilde{U}^{\prime}+\left(I-\tilde{U} \tilde{U}^{\prime}\right) N^{-1 / 2} Q X Q N^{-1 / 2}\left(I-\tilde{U} \tilde{U}^{\prime}\right)\right\} N^{1 / 2},
\end{gathered}
$$

and

$$
N^{1 / 2}\left(Q^{-1} A+A^{\prime} Q^{-1}+Q^{-1} G G^{\prime} Q^{-1}\right) N^{1 / 2}=\tilde{U} \tilde{\Xi} \tilde{U}^{\prime} .
$$

(Proof) It is clear that (55) and (56) are equivalent to (26) and (53), respectively. We can obtain (54) as follows. Since we have from (53)

$$
Q^{-1} A+A^{\prime} Q^{-1}+Q^{-1} G G^{\prime} Q^{-1}=N^{-1 / 2} \tilde{U} \tilde{\Xi} \tilde{U}^{\prime} N^{-1 / 2},
$$

it is seen from (25) that

$$
\begin{aligned}
F & =Q\left\{Q^{-1} A-N^{-1 / 2} \tilde{U} \tilde{\Xi} \tilde{U}^{\prime} N^{-1 / 2}\right\} \\
& =-Q\left(A^{\prime}+Q^{-1} G G^{\prime}\right) Q^{-1} .
\end{aligned}
$$

Substitution of (58) into (27) yields

$$
-Q^{-1}\left(A^{\prime}+Q^{-1} G G^{\prime}\right)^{\prime} Q X-X Q\left(A^{\prime}+Q^{-1} G G^{\prime}\right) Q^{-1}+M=0,
$$

from which we obtain (54) by multiplications of $Q$ from both left and right. This completes the proof.

The next step is to solve (54)-(56) under (22) and (14). In order to make it easy to do this, let us introduce a new variable $W \in \boldsymbol{R}^{n \times n}$ by

$$
W \triangleq Q X Q .
$$

Then, we have the following set of equations for $(Q, \tilde{U}, \tilde{\Xi}, W)$ from (54)-(56).

$$
\begin{gathered}
\left(A+G G^{\prime} Q^{-1}\right) W+W\left(A+G G^{\prime} Q^{-1}\right)^{\prime}-Q M Q=0, \\
W=N^{1 / 2}\left\{\tilde{U} \tilde{\Psi} \tilde{U}^{\prime}+\left(I-\tilde{U} \tilde{U}^{\prime}\right) N^{-1 / 2} W N^{-1 / 2}\left(I-\tilde{U} \tilde{U}^{\prime}\right)\right\} N^{1 / 2},
\end{gathered}
$$

and

$$
N^{1 / 2}\left(Q^{-1} A+A^{\prime} Q^{-1}+Q^{-1} G G^{\prime} Q^{-1}\right) N^{1 / 2}=\tilde{U} \tilde{\Xi} \tilde{U}^{\prime} .
$$

[Remark 5] It should be noted that we have the following mutual dependences of the components of the solution $(Q, \tilde{U}, \tilde{\Xi}, W)$ of (61), (62) and (56).

(i) When true value of $W$ is known, $Q$ is determined by 
the following equation with Lyapunov-like linear terms with respect to $Q^{-1}$.

$$
\begin{aligned}
& W^{-1} G G^{\prime} Q^{-1}+Q^{-1} G G^{\prime} W^{-1} \\
& -\left(W^{-1} Q M Q W^{-1}-W^{-1} A-A^{\prime} W^{-1}\right)=0,
\end{aligned}
$$

(ii) When true value of $Q$ is known, $W$ is determined by the Lyapunov equation (61).

(iii) When true value of $\tilde{U}$ is known, $W$ must satisfy (62).

(iv) When true value of $Q$ is known, $(\tilde{U}, \tilde{\Xi})$ is determined by (56) as the eigenvectors and eigenvalues of the left-hand side.

(v) When true value of $(\tilde{U}, \tilde{\Xi})$ is known, $Q$ is determined by the Riccati equation (53).

We can easily see the following properties for (63).

«Theorem 5» Assume (C-1)-(C-3). For any positive definite symmetric matrix $W \in \boldsymbol{R}^{n \times n}$, let

$$
\mathscr{S}^{+}(A, M, W) \triangleq\left\{Q \in \boldsymbol{R}^{n \times n} ; Q M Q-A W-W A^{\prime}>0, Q^{\prime}=Q\right\},
$$

and

$$
\mathscr{S}^{-}(A, M, W) \triangleq\left\{Q \in \boldsymbol{R}^{n \times n} ; Q M Q-A W-W A^{\prime}<0, Q^{\prime}=Q\right\},
$$

Then, we have the following properties for $\mathscr{S}^{+}(A, M, W)$ and $\mathscr{S}^{-}(A, M, W)$.

(a) For the case $A W+W A^{\prime}<0, \quad \mathscr{S}^{+}(A, M, W)=$ $\left\{Q \in \boldsymbol{R}^{n \times n} ; Q^{\prime}=Q\right\}$ and $\mathscr{S}^{-}(A, M, W)=\phi$.

(b) For the case $A W+W A^{\prime}>0,0 \in \mathscr{S}^{-}(A, M, W)$.

(c) If $Q \in \mathscr{S}^{+}(A, M, W)$, then $-Q \in \mathscr{S}^{+}(A, M, W)$.

(d) If $Q \in \mathscr{S}^{-}(A, M, W)$, then $-Q \in \mathscr{S}^{-}(A, M, W)$.

Also, we have the following properties of the solution $Q$ of (63).

(i) When (63) has a solution $Q$ in $\mathscr{S}^{+}(A, M, W)$, it is positive-definite, i.e., $Q>0$.

(ii) When (63) has a solution $Q$ in $\mathscr{S}^{-}(A, M, W)$, it is negative-definite, i.e., $Q<0$.

(Proof) The properties (a)-(d) are clear by (64) and (65). Next, let us show (i). For any $\tilde{Q} \in \mathscr{P}^{+}(A, M, W)$, the Lyapunov equation of $\hat{Q}^{-1}$ :

$$
\begin{aligned}
& W^{-1} G G^{\prime} \hat{Q}^{-1}+\hat{Q}^{-1} G G^{\prime} W^{-1} \\
& -\left(W^{-1} \tilde{Q} M \tilde{Q} W^{-1}-W^{-1} A-A^{\prime} W^{-1}\right)=0,
\end{aligned}
$$

has a positive-definite and symmetric solution $\hat{Q}^{-1}$. Thus, we have a unique positive-definite symmetric matrix $\hat{Q}=\hat{Q}(\tilde{Q})$ for any $\tilde{Q} \in \mathscr{S}^{+}(A, M, W)$. If (63) has a solution $Q$ in $\mathscr{S}^{+}(A, M, W)$, then we must have $Q=\hat{Q}(Q)$. Since $\hat{Q}(Q)$ in the right-hand side is positive-definite and symmetric, $Q$ is a positive-definite symmetric matrix. Similarly, we can show (ii) as follows. For any $\tilde{Q} \in \mathscr{S}^{-}(A, M, W)$, the solution $\hat{Q}^{-1}$ of (66) is a negative-definite symmetric matrix. Hence, if (63) has a solution $Q$ in $\mathscr{S}^{-}(A, M, W)$, then we must have $Q=\hat{Q}(Q)$ which implies that $Q$ is a negative-definite symmetric matrix. This completes the proof.

In closing this section, two special cases are described in each of which the solution $(Q, \tilde{U}, \tilde{\Xi}, W)$ of $(61),(62)$ and (56) is obtained much easier than in the general case.

[Case 1: $\tilde{m}=m=n$ and $\tilde{\Psi}=I$ ] For this case, since $\tilde{U}=U$ is an orthogonal matrix, we have $\tilde{U} \tilde{U}^{\prime}=\tilde{U}^{\prime} \tilde{U}=I$. Hence, (62) is reduced to

$$
W=N
$$

Namely, $W$ is a known constant in this case. Then, (63) becomes

$$
\begin{aligned}
& N^{-1} G G^{\prime} Q^{-1}+Q^{-1} G G^{\prime} N^{-1} \\
& -\left(N^{-1} Q M Q N^{-1}-N^{-1} A-A^{\prime} N^{-1}\right)=0,
\end{aligned}
$$

which is a matrix equation with respect to only single variable $Q$. If we obtain solution $Q$ of (68), then, $(\tilde{U}, \tilde{\Xi})$ is determined by (56) and we have a complete solution of Problem 2.

[Case 2: $\tilde{m}=m=n$ ] In this case, $\tilde{\Psi}$ is any diagonal matrix with property (18). For this case, we also have $\tilde{U} \tilde{U}^{\prime}=\tilde{U}^{\prime} \tilde{U}=I$. However, (62) is not reduced to (67) but to

$$
W=N^{1 / 2} \tilde{U} \tilde{\Psi} \tilde{U}^{\prime} N^{1 / 2} .
$$

Therefore, we have to solve the set of equations: (63), (69) and (56) in order to obtain a solution $(Q, \tilde{U}, \tilde{\Xi}, W)$ of Problem 2.

The solutions $(Q, \tilde{U}, \tilde{\Xi}, W)$ of Problem 2 for the above two special cases are easily obtained by the recursive algorithms which will be given in the next section.

\section{Numerical Algorithms and Examples}

In this section, we propose recursive algorithms for the two special cases: Case 1 and Case 2. Using these algorithms, we show the results of numerical calculations for simple examples. Let us start with noting that equation (63) is obtained as the steady-state relation of the following matrix differential equation.

$$
\left\{\begin{aligned}
\frac{d Q^{-1}(t)}{d t} & =-N^{-1} G G^{\prime} Q^{-1}(t)-Q^{-1}(t) G G^{\prime} N^{-1} \\
& +N^{-1} Q(t) M Q(t) N^{-1}-N^{-1} A-A^{\prime} N^{-1} \\
Q^{-1}(0) & =Q_{0}^{-1}
\end{aligned}\right.
$$

Hence, we can compute the solution of (63) by the following algorithm.

[Algorithm for Case 1: $\tilde{m}=m=n$ and $\tilde{\Psi}=I$ ]

(i) Take any initial value $Q_{0}>0$ such that

$$
Q_{0}>M^{-1 / 2}\left\{M^{1 / 2}\left(A N+N A^{\prime}\right) M^{1 / 2}\right\}^{1 / 2} M^{-1 / 2} .
$$

Let $i=0$. 
(ii) Update $Q_{i}^{-1}$ and $Q_{i}$ by the recursive equation:

$$
\left\{\begin{aligned}
\Delta Q_{i}^{-1}= & \left(-N^{-1} G G^{\prime} Q_{i}^{-1}-Q_{i}^{-1} G G^{\prime} N^{-1}\right. \\
& \left.+N^{-1} Q_{i} M Q_{i} N^{-1}-N^{-1} A-A^{\prime} N^{-1}\right) \Delta t, \\
Q_{i+1}^{-1}= & Q_{i}^{-1}+\Delta Q_{i}^{-1}, \\
Q_{i+1}= & \left(Q_{i+1}^{-1}\right)^{-1},
\end{aligned}\right.
$$

(iii) (a) If $Q_{i+1} \cong Q_{i}$, then set as $Q=Q_{i+1}$. Go to (iv).

(b) Otherwise, let $i+1 \rightarrow i$ and return to (ii).

(iv) Compute $(\tilde{U}, \tilde{\Xi})$ by (56).

(v) Compute $H$ by (19).

We can also apply the above recursive computation (i)-(iii) to Case 2 as follows.

[Algorithm for Case 2: $\tilde{m}=m=n$ ]

(i) Take any initial value $Q_{00}>0$ such that

$$
Q_{00}>M^{-1 / 2}\left\{M^{1 / 2}\left(A N+N A^{\prime}\right) M^{1 / 2}\right\}^{1 / 2} M^{-1 / 2} .
$$

Also, take initial values as $\tilde{U}_{0}=I, W_{0}=N^{1 / 2} \tilde{\Psi} N^{1 / 2}$ and $W_{0}^{-1}=N^{-1 / 2} \tilde{\Psi}^{-1} N^{-1 / 2}$. Let $i=0$ and $k=0$.

(ii) Update $Q_{k i}^{-1}$ and $Q_{k i}$ by the recursive equation

$$
\left\{\begin{aligned}
\Delta Q_{k i}^{-1}= & \left(-W_{k}^{-1} G G^{\prime} Q_{i}^{-1}-Q_{i}^{-1} G G^{\prime} W_{k}^{-1}\right. \\
& \left.+W_{k}^{-1} Q_{i} M Q_{i} W_{k}^{-1}-W_{k}^{-1} A-A^{\prime} W_{k}^{-1}\right) \Delta t, \\
Q_{k i+1}^{-1}= & Q_{k i}^{-1}+\Delta Q_{k i}^{-1}, \\
Q_{k i+1}= & \left(Q_{k i+1}^{-1}\right)^{-1},
\end{aligned}\right.
$$

(iii) (a) If $Q_{k i+1} \cong Q_{k i}$, then set as $Q_{k}=Q_{k i+1}$. Go to (iv).

(b) Otherwise, let $i+1 \rightarrow i$ and return to (ii).

(iv) Update $\left(\tilde{U}_{k}, \tilde{\Xi}_{k}\right)$ by

$$
\begin{aligned}
& N^{1 / 2}\left(Q_{k}^{-1} A+A^{\prime} Q_{k}^{-1}+Q_{k}^{-1} G G^{\prime} Q_{k}^{-1}\right) N^{1 / 2} \\
& =\tilde{U}_{k+1} \tilde{\Xi}_{k+1} \tilde{U}_{k+1}^{\prime},
\end{aligned}
$$

(v) (a) If $\left(\tilde{U}_{k+1}, \tilde{\Xi}_{k+1}\right) \cong\left(\tilde{U}_{k}, \tilde{\Xi}_{k}\right)$, then set as $Q=Q_{k}$ and $(\tilde{U}, \tilde{\Xi}) \cong\left(\tilde{U}_{k+1}, \tilde{\Xi}_{k+1}\right)$ go to (vi).

(b) Otherwise, let $i=0$ and $k+1 \rightarrow k$, and set as $Q_{k 0}=Q_{k-1}$. Return to (ii).

(vi) Compute $H$ by (19).

In the remainder of this section, we will give illustrative examples of 3-dimensional systems $(n=m=\tilde{m}=3)$ for each of the above two algorithms.

Example 1 (Case 1). Let us consider a 3-dimensional system with

$$
\begin{gathered}
A=\left[\begin{array}{rrr}
-0.10 & -0.03 & 0.02 \\
0.02 & -0.10 & 0.01 \\
0.06 & 0.03 & -0.08
\end{array}\right], \quad G=\left[\begin{array}{ccc}
0.5 & 0 & 0 \\
0 & 0.5 & 0 \\
0 & 0 & 0.5
\end{array}\right], \\
R_{0}=I, \quad M=\left[\begin{array}{rrr}
14.460 & 16.764 & 9.3614 \\
16.764 & 33.371 & 25.688 \\
9.3614 & 25.688 & 24.090
\end{array}\right], \quad N=0.5 I .
\end{gathered}
$$

By taking $Q_{00}=0.3 \cdot I$ and $\Delta t=0.05$, we have made numerical computations for the case $\tilde{m}=3$.

The result of the algorithm for Case 1 is summarized in Table 1.

Example 2 (Case 2). Let us consider the same system as

\begin{tabular}{|c|c|c|}
\hline Variable & \multicolumn{2}{|c|}{ Optimal Value } \\
\hline$Q$ & {$\left[\begin{array}{rr}0.33943 & -0.13832 \\
-0.13832 & 0.37230 \\
0.05861 & -0.18512\end{array}\right.$} & $\left.\begin{array}{r}0.05861 \\
-0.18512 \\
0.35447\end{array}\right]$ \\
\hline$U$ & {$\left[\begin{array}{rr}0.38314 & 0.82688 \\
0.71215 & 0.01939 \\
0.58825 & -0.56204\end{array}\right.$} & $\left.\begin{array}{r}0.41166 \\
-0.70176 \\
0.58144\end{array}\right]$ \\
\hline$\Xi$ & \multicolumn{2}{|c|}{$\operatorname{diag}\{4.68949,1.04112,0.18284\}$} \\
\hline$N^{1 / 2} H^{\prime} R_{0}^{-1} H N^{1 / 2}$ & {$\left[\begin{array}{ll}1.43124 & 1.24342 \\
1.24342 & 2.46875 \\
0.61686 & 1.87860\end{array}\right.$} & $\left.\begin{array}{l}0.61686 \\
1.87860 \\
2.01345\end{array}\right]$ \\
\hline$H^{\prime} R_{0}^{-1} H$ & {$\left[\begin{array}{ll}2.86249 & 2.48685 \\
2.48685 & 4.93750 \\
1.23371 & 3.75719\end{array}\right.$} & $\left.\begin{array}{l}1.23371 \\
3.75719 \\
4.02691\end{array}\right]$ \\
\hline$H$ & {$\left[\begin{array}{rr}1.32265 & 2.46100 \\
1.08739 & 0.19442 \\
-0.12767 & -0.60419\end{array}\right.$} & $\left.\begin{array}{r}1.92658 \\
-0.73227 \\
0.27771\end{array}\right]$ \\
\hline$J$ & \multicolumn{2}{|c|}{16.42288068} \\
\hline
\end{tabular}
in Example 1 with $R_{0}$ replaced by

$$
\begin{aligned}
R_{0} & =\left[\begin{array}{ccc}
-\frac{1}{8} & -\frac{3 \sqrt{3}}{8} & \frac{3}{4} \\
-\frac{3 \sqrt{3}}{8} & \frac{5}{8} & \frac{\sqrt{3}}{4} \\
-\frac{3}{4} & -\frac{\sqrt{3}}{4} & -\frac{1}{2}
\end{array}\right]\left[\begin{array}{ccc}
0.49 & 0 & 0 \\
0 & 1.0 & 0 \\
0 & 0 & 1.44
\end{array}\right]\left[\begin{array}{ccc}
-\frac{1}{8} & -\frac{3 \sqrt{3}}{8} & -\frac{3}{4} \\
-\frac{3 \sqrt{3}}{8} & \frac{5}{8} & -\frac{\sqrt{3}}{4} \\
\frac{3}{4} & \frac{\sqrt{3}}{4} & -\frac{1}{2}
\end{array}\right] \\
& =\left[\begin{array}{rrr}
1.2395 & 0.1015 & -0.2128 \\
0.1015 & 0.8673 & -0.3437 \\
-0.2128 & -0.3437 & 0.8231
\end{array}\right],
\end{aligned}
$$

i.e.,

$$
\Psi=\operatorname{diag}(0.49,1.0,1.44), \quad \Gamma=\left[\begin{array}{ccc}
-\frac{1}{8} & -\frac{3 \sqrt{3}}{8} & \frac{3}{4} \\
-\frac{3 \sqrt{3}}{8} & \frac{5}{8} & \frac{\sqrt{3}}{4} \\
-\frac{3}{4} & -\frac{\sqrt{3}}{4} & -\frac{1}{2}
\end{array}\right] .
$$

By taking $Q_{00}=I$ and $\Delta t=0.05$, we have made numerical computations for the case $\tilde{m}=3$.

The result of the algorithm for Case 2 is summarized in Table 2.

Table 1. The result by the proposed algorithm (Case 1)

It is rather surprising and should be noted that the values of $\tilde{U}=U$ are almost same for the both cases although the values of other variables are considerably different from each other. This result is of both theoretical and applicational interest because the solution of this optimization problem is obtained very easily if this is true for all or a large class of cases. 
Table 2. The result by the proposed algorithm (Case 2)

\begin{tabular}{|c|cr|}
\hline Variable & Optimal Value \\
\hline \multirow{4}{*}{$Q$} & {$\left[\begin{array}{rrr}0.34865 & -0.16870 & 0.06961 \\
-0.16870 & 0.39022 & -0.22813 \\
0.06961 & -0.22813 & 0.36711\end{array}\right]$} \\
\hline \multirow{*}{*}{$\Xi$} & {$\left[\begin{array}{rrr}0.38059 & 0.82764 & 0.41250 \\
0.71515 & 0.01937 & -0.69870 \\
0.58626 & -0.56092 & 0.58452\end{array}\right]$} \\
\hline \multirow{2}{*}{$W$} & $\operatorname{diag}\left\{\begin{array}{rrr}0.63430,1.04069,0.13293\} \\
-0.13281 & 0.47698 & -0.19676 \\
-0.00385 & -0.19676 & 0.48752\end{array}\right]$ \\
\hline$N^{1 / 2} H^{\prime} R_{0}^{-1} H N^{1 / 2}$ & {$\left[\begin{array}{rrr}1.84132 & 2.05629 & 1.25235 \\
2.05629 & 3.96979 & 3.13522 \\
1.25235 & 3.13522 & 2.99680\end{array}\right]$} \\
\hline$H^{\prime} R_{0}^{-1} H$ & {$\left[\begin{array}{rrr}3.68264 & 4.11259 & 2.50469 \\
4.11259 & 7.93958 & 6.27044 \\
2.50469 & 6.27044 & 5.99360\end{array}\right]$} \\
\hline$H^{-1} H$ & {$\left[\begin{array}{rrr}-1.62097 & -3.05745 & -2.60174 \\
0.98703 & -0.01974 & -0.88726 \\
0.11460 & -0.03459 & 0.65514\end{array}\right]$} \\
\hline & 15.80750369 \\
\hline
\end{tabular}

\section{Conclusions}

In this paper, we considered the optimization problem of the observation gain matrix for the stationary Kalman-Bucy filter. The optimal gain matrix is obtained from the eigenvalues and the eigenvectors of SSNRM which is a symmetric matrix of a quadratic function of the gain matrix and is linearly dependent on the inverse of the observation noise covariance matrix. The condition of optimality has been obtained, and it has been shown that this condition is the same as the one which was obtained in the corresponding discrete-time problem except for the point that the accompanying equation is a continuous-type Lyapunov equation whereas it was a discrete-type Lyapunov equation in the latter problem. This shows the condition of optimality has the universal validity both for continuous- and discrete-time systems. The solution of a set of equations, the matrix Riccati equation for the error covariance matrix and those of the condition of optimality, can be solved by introducing a simple recursive algorithm.

\section{References}

[1] M. Athans, "On the determination of optimal costly measurement strategies for linear stochastic systems," Prep. 5th IFAC World Congress, Paris, 1/11, 1972.

[2] A. Logothetis, A. Isaksson and E. Skadas, "On sensor scheduling via information theoretic criteria," Prep. ACC, 1999.
[3] M. Aoki, and M. T. Li, "Optimal discrete time control system with cost for observation," IEEE Trans. Automatic Control, AC-14, 2, 165/175, 1969.

[4] A. Sano and M. Terao, "Measurement optimization in optimal process control," Automatica, 5, 705/714, 1970.

[5] S. Ihara, "Coding theory in white Gaussian channel with feedback," J. Multi-variate Analysis, 4, 74/87, 1974.

[6] Y. Takeuchi, "The optimal transmission of non-Gaussian signals through a noisy channel with feedback," IEEE Trans. Inform. Theory, IT-40, 5, 1624/1629, 1994.

[7] Y. Takeuchi and A. Ida, "Optimal Transmission of a set of Gaussian Signals through Discrete-Time Channels with Feedback," Proc. of 35th ISCIE SSS, Ube, Oct. 30-31, 2003, 118/123, 2004.

[8] Y. Takeuchi, "An information theoretic approach to optimization of linear observations for the Kalman-Bucy filter," International Journal of Innovative Computing, Information \& Control, 1-3, 401/416, 2005.

[9] Y. Takeuchi, "Optimal transmission of a set of discrete-time Gaussian signals through channels with feedback," International Journal of Innovative Computing, Information \& Control, 2-5, 927/942, 2006.

[10] Y. Takeuchi, "Optimization of linear observations for the stationary Kalman filter based on a generalized Water Filling Theorem," International Journal of Innovative Computing, Information \& Control, 4-1, 211/230, 2008.

[11] Y. Takeuchi and A. Hirata, "A method of optimization of linear observations for the Kalman filter based on a generalized Water Filling Theorem," International Journal of Innovative Computing, Information \& Control, 5-1, 75/85, 2009.

[12] Y. Takeuchi and M Inoue, "Optimization of observations for LQG control systems by an information theoretic approach," International Journal of Innovative Computing, Information \& Control, 6-1, 75/87, 2010.

[13] Y. Takeuchi, "Optimization of linear observations for the stationary Kalman filter under a quadratic performance criterion," International Journal of Innovative Computing, Information \& Control, 7-1, 85/99, 2011.

[14] Y. Takeuchi, "Optimization of the observations for stationary LQG stochastic control systems under a quadratic criterion," International Journal of Innovative Computing, Information \& Control, 8-3, 2313/2328, 2012.

[15] Y. Takeuchi and R. Matsumi, "Hybrid optimization of the observations for stationary LQG control systems," Trans. ISCIE, 25-12, 366/374, 2012.

[16] Y. Takeuchi and H. Nakai, "A New Method of Optimization of Linear Observations for the Stationary Kalman Filter," Proc. of 44th ISCIE SSS, Tokyo, Nov. 1-2, 2012, 21/28, 2013.

[17] Y. Takeuchi and H. Nakai, "An Improved Method of Optimization of Linear Observations for the Stationary Kalman Filter," Proc. of 45th ISCIE SSS, Okinawa, Nov. 1-2, 2013, 1/8, 2014.

[18] S. Kodama, and N. Suda, Matrix Theory for Systems Control (in Japanese), SICE, 1978.

[19] V. Kučera, "A contribution to matrix quadratic equations," IEEE Trans. Automatic Control, AC-17, 344/347, 1972. 